PROC. OF JSCE,

No. 212, APRIL, 1973

\title{
ANISOTROPY AND STRESS DISTRIBUTION IN SAND
}

\author{
By Nobuchika MOROTO*
}

\section{SYNOPSIS}

Sand has both inherent and induced anisotropy. The former is produced by sedimentation and the latter occurs in shear. Degree of the anisotropy is evaluated by means of the deformation modulus ratio $n=E_{x} / E_{z}$, and the case $n<1$ is treated.

Deriving the solutions of stress and displacement in a cross-anisotropic soil, effect of $n$ on the stress and surface settlement in sand is examined. The results show that the stress and surface settlement tend to increase near the load in case of $n<1$. This stress increase in plane strain case is smaller than that in axial symmetry. It can be known that the author's stress solution is not coincidence with Wolf's.

From the evidence that a radial stress field is formed in the plane strain case, it can be considered that the value $n$ will be partly responsible for the measured concentration index in Frölich's formula.

Usefulness of the anisotropic stress-strain laws for expressing the dilatancy of soil is emphasized.

\section{INTRODUCTION}

Two types of anisotropy have been observed in sand, namely, an inherent anisotropy and an induced anisotropy. The inherent anisotropy is produced by sedimentation through water or pouring in air. The induced anisotropy occurs in successive shearing process. Such anisotropies will have an influence on stresses in sand and displacement of foundation resting on it. It has been known that the vertical stress in loaded sand mass exceeds the indicated value by Boussinesq's equation. Investigators have studied this problem theoretically (Ohde 1936, Borowicka 1943) and in experiments (Ichihara 1955, Curtis \& Richard 1955, Turnbull et al. 1961). The devi-

* Grad. Student, Department of Civil Engineering Tohoku University, Sendai ation of the stress in sand from that in the ideal elastic media has been explained by pointing out the following main reasons, (1) the sand adjacent to the loaded area at the surface tends to yield even by low stress, (2) the rigidity of sand increases with depth. The author considers, however, the anisotropies of sand may also contribute to the measured stresses and displacement in sand. Barden (1963) has made a short remark on an effect of anisotropy on the behaviour of sand mass.

Nonlinearity and anisotropy in the behaviour of sand are evident. Hasegawa and Sawada (1971) show, in their paper of stress solutions in nonlinear and heterogeneous elastic half space, that problem of material nonlinearity can be treated as a kind of problem where rigidity varies with depth. Then, to evaluate the anisotropic effect on stress distribution in sand in a simple manner, in this paper, the linear stress-strain laws are applied.

\section{SOME ANISOTROPIC FEATURES OF SAND}

Kallstenius and Bergan (1961) showed that even spherical particle tended to pack anisotropically in deposition. Oda (1970) fixed sand particles with polyester resin to examine the initial fabrics of sand sample prepared by sedimentation in water. He stated that the particles deposited in this way to adopt a preferred orientation, with the maximum dimension alined in horizontal plane. Parkin et al. (1968) reported that the lateral strain of the triaxial sample of sand was much greater than the vertical strain in isotropic compression as shown in Fig. 1. Their tests were carried out on medium sand deposited in air. El-Shoby conducted a series of constant stress ratio tests on fine sand. His figure shows the greater lateral deformability.

Moroto (1972) carried out the repetitional loading tests on a river sand under an isotropic stress condition. He reported that the triaxial sand specimen became almost isotropic. Karst et al. 


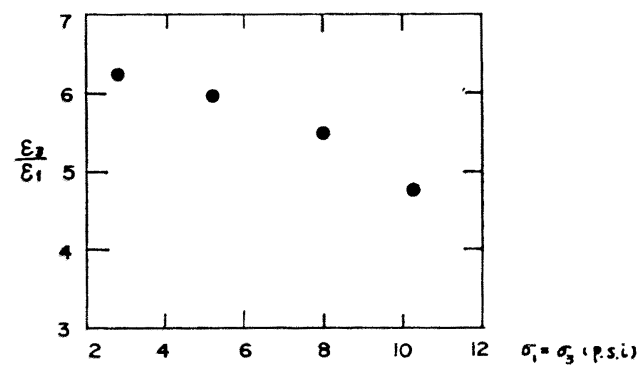

Fig. 1 Variation of Strain Ratio in Isotropic Compression (After Parkin, et al.).

(1961) also noted that a reduction of initial anisotropy occured with repititions of isotropic load.

Rowe (1962) has shown that the ratio of number of points of contact per unit horizontal area to that of vertical area depends on the ratio of applied stress and is greater than unity. Oda (1972) stated in his study on the fabrics of sand sample in loading that a preferred re-orientation of the initial fablics occurs with increase of axial strain, and that the long axis of grains tended to aline in the direction perpendicular to the axial stress. According to this the sand sample becomes anisotropic. The anisotropy in shear were related to the dilatancy as follow by Oda,

$$
\frac{d v}{d \varepsilon_{a}}=k_{3} \frac{S_{z}}{S_{x}}-k_{4}
$$

where $S_{z}$ and $S_{x}$ are summations of the projected area of contact surface at the contacting point of sand particles on the horizontal and vertical planes respectively. $d v$ is the volumetric strain increment and $d \varepsilon_{a}$ is the axial strain increment. $k_{3}$ and $k_{4}$ are constants determined from test. The ratio $S_{x} / S_{z}$ is reported less than unity in the paper of Oda.

Such structures of sand sample should evidently be conductive to the greater lateral deformability. Barden (1963) wrote, "Current unpublished work at Manchester University, both theoretical and experimental, indicates that for sands $n$ (the ratio of lateral stiffness to vertical stiffness) can be considerably less than unity."

In principle, the case $n>1$ can be considered and probably exists. As has been seen, however, it can be recognized that the case $n<1$ is commonly encountered for sand. The case $n<1$ is therefore interesting in this paper.

\section{STRESS-STRAIN LAWS FOR DILA- TANT ANISOTROPIC SOIL}

Granular material dilates in shear. Stress- strain laws including a term of the volume change due to shear have been therefore required. Isotropic formulae are inappropriate for the purpose. Considering the experimental facts that sand shows anisotropic natures in deformation, let anisotropic stress-strain relations be applied here.

Axial symmetric case

$$
\left(E_{x}=E_{y}=E_{r}, \quad \sigma_{x}=\sigma_{y}=\sigma_{r}, \quad \varepsilon_{x}=\varepsilon_{y}=\varepsilon_{r}\right)
$$

Write the stress-strain relations of triaxial sample as follows in a conventional form,

$$
\begin{aligned}
& \varepsilon_{r}=\frac{1-\nu_{r r}}{E_{r}} \sigma_{r}-\frac{\nu_{a r}}{E_{a}} \sigma_{a} \\
& \varepsilon_{a}=-\frac{2 \nu_{r a}}{E_{r}} \sigma_{r}+\frac{1}{E_{a}} \sigma_{a} .
\end{aligned}
$$

It has been justified that mechanical behaviour of soil are better expressed by the spherical and deviatric components of stress tensor. Then, rewrite the above formulae as

$$
\begin{aligned}
& v=\varepsilon_{a}+2 \varepsilon_{r}=C \cdot p-D \cdot q \\
& \gamma=\varepsilon_{a}-\varepsilon_{r}=A \cdot p+B \cdot q .
\end{aligned}
$$

where

$$
\begin{aligned}
& p=\frac{1}{3}\left(\sigma_{a}+2 \sigma_{r}\right) \\
& q=\frac{1}{3}\left(\sigma_{a}-\sigma_{r}\right)
\end{aligned}
$$

and $C, D, A, B$ are constants.

From Eqs. (2), (3) and (4), isotropic compression gives the constant $C$ as,

$$
C=\frac{1}{E_{a}}+\frac{2}{E_{r}}-\left(\frac{2 \nu_{a r}}{E_{a}}+\frac{2 \nu_{r a}}{E_{r}}+\frac{2 \nu_{r r}}{E_{r}}\right) \ldots
$$

Substituting Eq. (8) into (4), the constant $D$ can be obtained

$$
D=2\left(\frac{1}{E_{r}}-\frac{1}{E_{a}}\right)+2\left(\frac{2 \nu_{a r}}{E_{a}}-\frac{\nu_{r a}}{E_{r}}-\frac{\nu_{r r}}{E_{r}}\right)
$$

The constants $C$ and $D$ are relating to the compressional behaviour and dilatancy respectively.

Comparing Eqs. (4), (5) with (2), (3), a set of constants in Eq. (4), (5) can be given,

$$
\begin{aligned}
& \frac{1}{E_{a}}=\{C-D+2(A+B)\} / 9 \\
& \frac{\nu_{r a}}{E_{r}}=\left\{B-\left(2 A+C+\frac{D}{2}\right)\right\} / 9 \\
& \frac{\nu_{a r}}{E_{a}}=\{B+A-C+D\} / 9 \quad \cdots \cdots \\
& \frac{1-\nu_{r r}}{E_{r}}=\{2 C+D-2 A+B\} / 9
\end{aligned}
$$

It is known that the conventional triaxial test is 
not sufficient to determine uniquely all the constants.

Plane strain case $\left(\varepsilon_{y}=0\right)$

Neglecting the second order terms with respect to the Poisson's ratios, write the stress-strain relations as,

$$
\begin{aligned}
& \varepsilon_{x}=\frac{1}{E_{x}} \sigma_{x}-\frac{\nu_{z x}}{E_{z}} \sigma_{z} \\
& \varepsilon_{z}=-\frac{\nu_{x z}}{E_{x}} \sigma_{x}+\frac{1}{E_{z}} \sigma_{z}
\end{aligned}
$$

Putting

$$
\begin{aligned}
& p=\frac{\sigma_{x}+\sigma_{z}}{2} \\
& q=\frac{\sigma_{z}-\sigma_{x}}{2}
\end{aligned}
$$

rewrite Eqs. (14), (15) as,

$$
\begin{aligned}
& v=\varepsilon_{x}+\varepsilon_{z}=\bar{C} p-\bar{D} q \\
& \gamma=\varepsilon_{z}-\varepsilon_{x}=\bar{A} p+\bar{B} q
\end{aligned}
$$

where $\bar{C}, \bar{D}, \bar{A}, \bar{B}$ are constants corresponding to $C, D, A, B$ respectively.

From Eqs. (18) and (14), (15), isotropic compression gives the constant $\bar{C}$ as,

$$
\bar{C}=\frac{1}{E_{x}}+\frac{1}{E_{z}}-\left(\frac{\nu_{x z}}{E_{x}}+\frac{\nu_{z x}}{E_{z}}\right)
$$

Substituting Eq. (20) into (18), the constant $\bar{D}$ becomes

$$
\bar{D}=\left(\frac{1}{E_{x}}-\frac{1}{E_{z}}\right)+\left(\frac{\nu_{z x}}{E_{z}}-\frac{\nu_{x z}}{E_{x}}\right)
$$

Comparing Eqs. (18), (19) with (14), (15), a set of constants in Eqs. (14), (15) can be written

$$
\begin{aligned}
& \frac{1}{E_{z}}=(\bar{C}-\bar{D}+\bar{A}+\bar{B}) / 4 \cdots \\
& \frac{\nu_{x z}}{E_{x}}=\{\bar{B}-(\bar{A}+\bar{C}+\bar{D})\} / 4 \\
& \frac{\nu_{z x}}{E_{z}}=\{\bar{B}+\bar{A}-\bar{C}+\bar{D}\} / 4 \\
& \frac{1}{E_{x}}=\{\bar{C}+\bar{D}-\bar{A}+\bar{B}\} / 4
\end{aligned}
$$

In Eqs. (11), (12) and (23), (24), the constants $A, D, \bar{A}, \bar{D}$ do not take naught for anisotropic and dilatant material. Hence,

$$
\begin{aligned}
& \frac{\nu_{a r}}{E_{a}} \neq \frac{\nu_{r a}}{E_{r}} \\
& \frac{\nu_{z x}}{E_{z}} \neq \frac{\nu_{x z}}{E_{x}}
\end{aligned}
$$

From these things, it is supposed that the restriction of $\frac{\nu_{a r}}{E_{a}}=\frac{\nu_{r a}}{E_{r}}$ or $\frac{\nu_{z x}}{E_{z}}=\frac{\nu_{x z}}{E_{x}}$ in the theory of elasticity does not hold in general for sand.

It can be known in Eqs. (9), (21) that the constants on the dilatancy, $D, \bar{D}$, can be expressed by the deformation moduli $E_{i}$ and Poisson's ratios $\nu_{i j}$. This shows the usefulness of the anisotropic formulae for dilatant anisotropic soil. Tests on sand necessarily bring errors and variations in the tested values of the moduli. In addition, accurate determination of Poisson's ratio is almost impossible at present. The attention shall be therefore focussed mainly on the deformation moduli $E_{a}, E_{r}$ or $E_{x}, E_{z}$ and their ratio $n=E_{r} / E_{a}$ or $n=E_{x} / E_{z}$.

Pickering (1970) discussed parameters in the linear elastic soil with a cross anisotropy. $\mathrm{He}$ used the relation $\frac{\nu_{z x}}{E_{z}}=\frac{\nu_{x z}}{E_{x}}$. In this case, from Eqs. (9) and (21), the constants $D, \bar{D}$ become as follows,

$$
\begin{aligned}
D & =2\left(\frac{1}{E_{r}}-\frac{1}{E_{a}}+\frac{\nu_{a r}}{E_{a}}-\frac{\nu_{r a}}{E_{r}}\right) \\
\bar{D} & =\frac{1}{E_{x}}-\frac{1}{E_{z}} \ldots \ldots \ldots \ldots \ldots \ldots \ldots \ldots \ldots \ldots \ldots
\end{aligned}
$$

Then, it can be known that the dilatancy is expressed mainly by the deformation moduli $E_{a}$ and $E_{r}$ in axial symmetric case and only by $E_{z}$ and $E_{x}$ in plane strain case. There is an objectionable matter given by relation (26) or (27) in the use of linear elasticity, though the linear elasticity is very interesting for soils engineering, because of its simplicity and convenience.

\section{STRESS AND DISPLACEMENT IN A CROSS ANISOTROPIC SOIL}

In order to evaluate the anisotropic effect on the stress and displacement in sand, a simple case of anisotropy is treated herein. Wolf (1935) is the first to consider the anisotropy of soil (to author's knowledge). However, the author has some questions about his solution in plane strain problem and the neglection of Poisson's ratios in

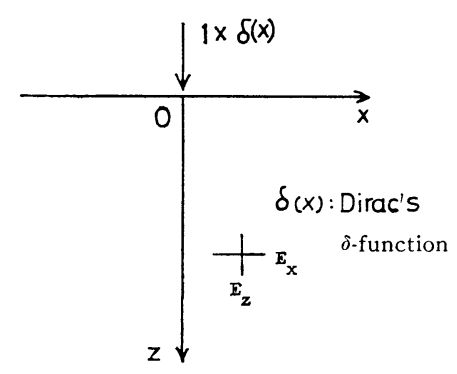

Fig. 2 Unit Line Load and Coordinates. 
three dimensional case.

First, stress and displacement solutions in a cross anisotropic soil shall be derived under plane strain condition. A system of Cartesian coordinates is taken and the $z$ - and $x$-directions are defined vertical and horizontal respectively. Consider the case of the principal axis of the material being coincidence with the coordinates axis.

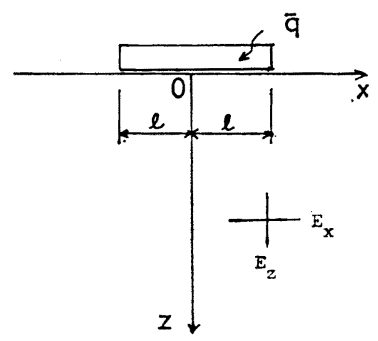

Fig. 3 Uniformly Distributed Load and Coordinates.

The stress-strain relations are written as follow by neglecting the second order terms with respect to Poisson's ratios,

$$
\begin{aligned}
& \varepsilon_{x}=\frac{1}{E_{x}} \sigma_{x}-\frac{\nu_{z x}}{E_{z}} \sigma_{z} \cdots . \\
& \varepsilon_{z}=-\frac{\nu_{x z}}{E_{x}} \sigma_{x}+\frac{1}{E_{z}} \sigma_{z} . . . \\
& \gamma_{x z}=\frac{1}{G_{x z}} \tau_{x z}
\end{aligned}
$$

The stresses can be expressed by Airy's stress function $F$ as,

$$
\sigma_{x}=\frac{\partial^{2} F}{\partial z^{2}}, \quad \sigma_{z}=\frac{\partial^{2} F}{\partial x^{2}}, \quad \tau_{x z}=-\frac{\partial^{2} F}{\partial x \partial z}
$$

The strain compatibility equation is

$$
\frac{\partial^{2} \varepsilon_{z}}{\partial x^{2}}+\frac{\partial^{2} \varepsilon_{x}}{\partial z^{2}}=\frac{\partial^{2} \gamma_{x z}}{\partial x \partial z}
$$

Substituting Eq. (33) into Eqs. (30) (32) and then into Eq. (34) gives

$$
\begin{aligned}
\frac{1}{E_{z}} \frac{\partial^{4} F}{\partial x^{4}} & +\left(\frac{1}{G_{x z}}-\frac{\nu_{x z}}{E_{x}}-\frac{\nu_{z x}}{E_{z}}\right) \frac{\partial^{4} F}{\partial x^{2} \partial z^{2}} \\
& +\frac{1}{E_{x}} \frac{\partial^{4} F}{\partial z^{4}}=0 \ldots \ldots \ldots \ldots \ldots \ldots \ldots
\end{aligned}
$$

Assuming $\frac{\nu_{z x}}{E_{z}}=\frac{\nu_{x z}}{E_{x}}$ and using the Barden's assumption of

$$
\frac{1}{G_{x z}}=\frac{1}{E_{z}}+\frac{1}{E_{x}}+\frac{2 \nu_{z x}}{E_{z}}
$$

the compatibility equation reduces to

$$
n \frac{\partial^{4} F}{\partial x^{4}}+(n+1) \frac{\partial^{4} F}{\partial x^{2} \partial z^{2}}+\frac{\partial^{4} F}{\partial z^{4}}=0
$$

where $n=\frac{E_{x}}{E_{z}}$.

Let the function be assumed as follow

$$
F=\int_{0}^{\infty} f(z, m) \cos m x d m
$$

where $m$ is a positive parameter. Substituting Eq. (38) into Eq. (37) gives

$$
\frac{d^{4} f}{d z^{4}}-(n+1) \frac{d^{2} f}{d z^{2}}+n=0
$$

The solution of the differential equation (39) becomes

$$
f=B_{1} e^{-r_{1} m z}+B_{2} e^{-r_{2} m z}
$$

where $\eta_{1}$ and $\eta_{2}$ are available characteristics roots which are given by

$$
\eta_{1}=\sqrt{n}=k, \quad \eta_{2}=1
$$

and $B_{1}, B_{2}$ are constants.

Substituting Eq. (40) into Eq. (33) gives

$$
\begin{aligned}
& \sigma_{z}=\frac{\partial^{2} F}{\partial x^{2}}=-\int_{0}^{\infty}\left(B_{1} e^{-r_{1} m z}+B_{2} e^{-\eta_{2} m z}\right) \\
& \times m^{2} \cos m x d m \\
& \sigma_{x}=\frac{\partial^{2} F}{\partial z^{2}}=\int_{0}^{\infty}\left(B_{1} \eta_{1}^{2} e^{-r_{1} m z}+B_{2} e^{-\eta_{2} m z}\right) \\
& \times m^{2} \cos m x d m \\
& \tau_{x z}=-\int_{0}^{\infty}\left(B_{1} \eta_{1} e^{-r_{1} m z}+B_{2} \eta_{2} e^{-r_{2} m z}\right) \\
& \times m^{2} \sin m x d m
\end{aligned}
$$

In the case that unit load is applied vertically at the origin of the coordinates, the boundary conditions are

$$
\begin{aligned}
& \left.\sigma_{z}\right|_{z=0}=1 \times \delta(x)=\frac{1}{\pi} \int_{0}^{\infty} \cos m x d m \\
& \left.\tau_{x z}\right|_{z=0}=0
\end{aligned}
$$

where $\delta(x)$ is Dirac's $\delta$-function.

Substituting Eq. (45) into Eq. (42) gives

$$
-\left(B_{1}+B_{2}\right) m^{2}=\frac{1}{\pi}
$$

Substituting Eq. (46) into Eq. (44) gives

$$
\eta_{1} B_{1}+\eta_{2} B_{2}=0
$$

Determining the constants $B_{1}$ and $B_{2}$ from Eqs. (47) and (48), and substituting them into Eqs. (42), (43) and (44) gives

$$
\begin{aligned}
& \left(\sigma_{z}, \sigma_{x}, \tau_{x z}\right)=\frac{\eta_{1} \eta_{2}\left(\eta_{1}+\eta_{2}\right)}{\pi} \frac{\left(z^{2}, x^{2}, x z\right) z}{R} . . \\
& R=\left\{\left(\eta_{1} z\right)^{2}+x^{2}\right\}\left\{\left(\eta_{2} z\right)^{2}+x^{2}\right\} \quad \ldots \ldots \ldots \ldots \ldots
\end{aligned}
$$

Substituting the relations of $\eta_{1}=k$ and $\eta_{2}=1$ into Eqs. (49) and (50) gives 


$$
\begin{aligned}
& \left(\sigma_{z}, \sigma_{x}, \tau_{x z}\right)=\frac{k(k+1)}{\pi \cdot R}\left(z^{2}, x^{2}, x z\right) z \ldots \ldots \ldots \\
& R=\left(z^{2}+x^{2}\right)\left\{(k z)^{2}+x^{2}\right\} \ldots \ldots \ldots \ldots \ldots \ldots \ldots \ldots \ldots \ldots \ldots \ldots \ldots
\end{aligned}
$$

On the other hand, Wolf's solution is

$$
\left(\sigma_{z}, \sigma_{x}, \tau_{x z}\right)=\frac{2 k}{\pi \cdot R}\left(z^{2}, x^{2}, x z\right) z
$$

Solution (51) does not coincide with Eq. (53) except only in case of unit $k$. The author considers that Wolf would make a mistake in determining the constant of his formula. The values of $k(1+k)$ and $2 k$ are calculated in Table 1 . This table shows the deviation between the author's stress solution and Wolf's.

Table 1 Values of $k(1+k)$ and $2 k$.

\begin{tabular}{c|c|c|c|c|c|c}
\hline$k$ & 1.0 & 0.9 & 0.8 & 0.7 & 0.6 & 0.5 \\
\hline$k(1+k)$ & 2.0 & 1.71 & 1.44 & 1.19 & 0.96 & 0.75 \\
\hline $2 k$ & 2.0 & 1.8 & 1.6 & 1.5 & 1.2 & 1.0 \\
\hline
\end{tabular}

The vertical displacement under unit line load can be obtained by integrating the following equation,

$$
\frac{\partial w}{\partial z}=\frac{1}{E_{z}}\left(1-\nu_{z x}^{2}\right) \sigma_{z}-\frac{\nu_{x z}}{E_{x}}
$$

as

$$
\begin{aligned}
w= & \frac{\eta_{1} \eta_{2}}{\pi\left(\eta_{2}-\eta_{1}\right)}\left[\frac{1}{E_{z}}\left(1-\nu_{z x}^{2}\right)\left(\frac{1}{2}\right) \ln \frac{\left\{\left(\eta_{1} z\right)^{2}+x^{2}\right\}^{1 / \eta_{1}}}{\left\{\left(\eta_{2} z\right)^{2}+x^{2}\right\}^{1 / \eta_{2}}{ }^{2}}\right. \\
& \left.-\frac{\nu_{x z}}{E_{x}}\left(\frac{1}{2}\right) \ln \frac{\left(\eta_{1} z\right)^{2}+x^{2}}{\left(\eta_{2} z\right)^{2}+x^{2}}\right]+C_{0} \quad \cdots \cdots \cdots(55)
\end{aligned}
$$

where $C_{0}$ is a constant of integration.

The surface settlement can be obtained by putting $z=0$ as,

$$
w_{0}=\frac{1}{\pi}\left(1+\frac{1}{k}\right) \frac{1-\nu_{z x}^{2}}{E_{z}} \ln |x|+C_{0}
$$

Putting the value of $w_{0}$ being zero at $x=x_{0}$ gives

$$
w_{0}=\frac{1}{\pi}\left(1+\frac{1}{k}\right) \frac{1-\nu_{z x}^{2}}{E_{z}} \ln \left|\frac{x}{x_{0}}\right|
$$

The stress and settlement formulae for uniformly distributed load can be obtained by integrating the solutions for line load.

Multiplying Eq. (51) by $\bar{q} \cdot d x$ and integrating from $(l-x)$ to $-(l+x)$ the stress formulae under uniformly distributed load are given by

$$
\begin{aligned}
\sigma_{z}= & \frac{\bar{q}}{\pi} \frac{1}{\eta_{2}-\eta_{1}}\left[\eta_{2} \tan ^{-1} \frac{2 l \eta_{1} z}{\left(\eta_{1} z\right)^{2}+x^{2}-l^{2}}\right. \\
& \left.-\eta_{1} \tan ^{-1} \frac{2 l \eta_{2} z}{\left(\eta_{2} z\right)^{2}+x^{2}-l^{2}}\right] \ldots \ldots \ldots \ldots \ldots \\
\sigma_{x}= & \frac{\bar{q}}{\pi} \frac{1}{\eta_{2}-\eta_{1}}\left[-\eta_{1} \tan ^{-1} \frac{2 \eta_{1} z}{\left(\eta_{1} z\right)^{2}+x^{2}-l^{2}}\right. \\
& \left.+\eta_{2} \tan ^{-1} \frac{2 l \eta_{2} z}{\left(\eta_{2} z\right)^{2}+x^{2}-l^{2}}\right] \ldots \ldots \ldots \ldots \ldots
\end{aligned}
$$

$$
\begin{aligned}
& \tau_{x z}=\frac{\bar{q}}{2 \pi} \frac{1}{\eta_{2}-\eta_{1}} \ln \left[\frac{(l+x)^{2}+\left(\eta_{1} z\right)^{2}}{(l+x)^{2}+\left(\eta_{2} z\right)^{2}}\right. \\
& \text { - } \left.\frac{(l-x)^{2}+\left(\eta_{2} z\right)^{2}}{(l-x)^{2}+\left(\eta_{1} z\right)^{2}}\right]
\end{aligned}
$$

It is easily recognized that Eqs. (58) (60) will be identical with those derived by Sobotoka (1964).

The surface settlement can be obtained by multiplying Eq. (57) by $\bar{q} \cdot d x$ and integrating from. $(l-x)$ to $-(l+x)$ as,

$$
w_{0}=\frac{1}{\pi}\left(1+\frac{1}{k}\right) \frac{1-\nu_{z x}^{2}}{E_{z}}\left[\ln \frac{\left|\frac{-x+l}{x_{0}}\right|^{|x-l|}}{\left|\frac{x+l}{x_{0}}\right|^{|x+l|}+2 l}\right]
$$

The vertical stress $\sigma_{z c}$ along the center line of the load and the surface settlement $w_{0 c}$ at the center of the load may be more interesting for engineering use.

Putting $x=0$ in Eq. (58) gives

$$
\sigma_{z c}=\frac{\bar{q}}{\pi} \frac{2 k}{1-k}\left[\tan ^{-1} \frac{(l / z)}{k}-k \tan ^{-1}(l / z)\right]
$$

Putting $z=0$ in Eq. (61) gives

$$
w_{0 c}=\frac{\bar{q}}{\pi}\left(1+\frac{1}{k}\right) \frac{1-\nu_{z x}^{2}}{E_{z}}(2 l) \text {. }
$$

Figs. 4 and 5 show the effect of $k$ on the vertical stress and the settlement due to strip load. It can be noted that an $n$ decreases so does the load spreading capacity of the medium, and both the vertical stress and the surface settle-

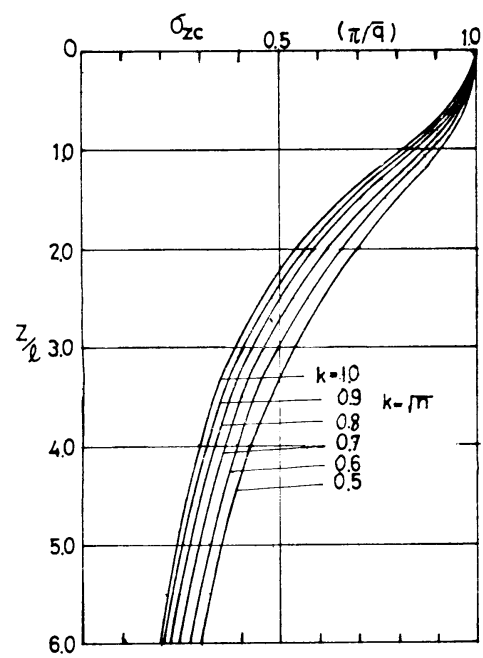

Fig. 4 Effect of Anisotropy on Stress Distributions. 
ment increase. The similar results have been shown by Barden for the three dimensional case.

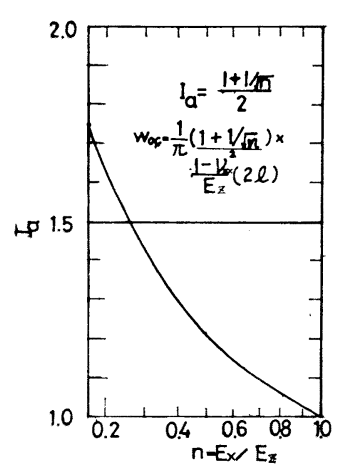

Fig. 5 Effect of Anisotropy on Surface Settlements.

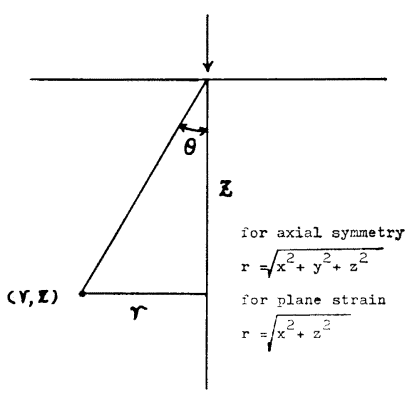

Fig. 6 Coordinates.

Barden modified Michell's solutions for a homogeneous cross-anisotropic elastic half space. With $r, \theta, z$ defined as in Fig. 6, he gave the stresses and settldments as follows,

For a vertical unit point load:

the vertical stress $\sigma_{z}$

$$
\sigma_{z}=h \cdot z
$$

the surface settlement $w_{0}$

$$
w_{0}=\frac{1}{2 \pi r} \sqrt{\frac{A}{G}} \cdot \frac{\left\{(\sqrt{A C}+G)^{2}-(F+G)\right\}^{1 / 2}}{A C-F^{2}}
$$

For a circular uniform load of radius $L$ with intensity $\bar{p}$ :

the vertical stress along the loading center line $\sigma_{z c}$

$$
\begin{gathered}
\sigma_{z c}=\bar{p}-\frac{\bar{p} \cdot z}{\sqrt{\alpha_{1}}-\sqrt{\alpha_{2}}}\left[\frac{1}{\left\{L^{2}+\frac{z^{2}}{\alpha_{1}}\right\}^{1 / 2}}\right. \\
\left.-\frac{1}{\left\{L^{2}+\frac{z^{2}}{\alpha_{2}}\right\}^{1 / 2}}\right] \ldots \ldots \ldots \ldots \ldots \ldots
\end{gathered}
$$

the surface settlement at the center of circular loaded area can be readily calculated as

$$
\begin{aligned}
w_{0} & =\int_{0}^{2 \pi} \int_{0}^{L} \bar{p} \cdot w_{0} \cdot r d r d \theta \\
& =\bar{p} L \sqrt{\frac{G}{A}} \cdot \frac{\left\{(\sqrt{A C}+G)^{2}-(F+G)^{2}\right\}^{1 / 2}}{A C-F^{2}}
\end{aligned}
$$

where

$$
\begin{aligned}
& h=\frac{1}{2 \pi} \cdot \frac{1}{\sqrt{\alpha_{1}}-\sqrt{\alpha_{2}}}\left[\frac{1}{\left\{r^{2}+\frac{z^{2}}{\alpha_{1}}\right\}^{3 / 2}}\right. \\
& \left.\quad-\frac{1}{\left\{r^{2}+\frac{z^{2}}{\alpha_{2}}\right\}^{3 / 2}}\right] \ldots \ldots \ldots \ldots \ldots \ldots \\
& \alpha_{1} \alpha_{2}=C / A, \quad \alpha_{1}+\alpha_{2}=\frac{A C-F^{2}-2 F G}{A C}
\end{aligned}
$$

and $A, C, G, F$ are defined by the following relations,

$$
\begin{aligned}
A & =\frac{n E_{z}}{\phi}\left(1-\nu_{z x} \nu_{x z}\right) \\
C & =\frac{E_{z}}{\phi}\left(1-\nu_{x y}^{2}\right) \ldots \ldots \ldots \ldots \ldots \ldots \ldots \ldots \ldots \ldots \ldots \ldots \ldots \ldots \ldots \ldots \ldots \ldots \\
F & =\frac{E_{z}}{\phi} \nu_{z x}\left(1+\nu_{x y}\right) \ldots \ldots \ldots \ldots \ldots \\
G & \fallingdotseq \frac{1}{E_{x}}+\frac{1}{E_{z}}+\frac{2 \nu_{z x}}{E_{z}} \ldots \ldots \ldots \ldots \\
\phi & =\left(1+\nu_{x y}\right)\left(1-\nu_{x y}-2 \nu_{x z} \nu_{z x}\right)
\end{aligned}
$$

\section{VERTICAL STRESS IN TWO AND THREE DIMENSIONS}

Compare the vertical stress in case of the plane strain with that in the axial symmetry.

Putting $x=0$ in Eq. (51), the vertical stress along the loading line for unit line load becomes

$$
\sigma_{z c}=\frac{1}{\pi}\left(1+\frac{1}{k}\right) \cdot \frac{1}{z}, \quad k=\sqrt{n}
$$

Putting $r=0$ in Eq. (64), the vertical stress along the loading axis for unit point load becomes

$$
\begin{aligned}
& \sigma_{z c}=h \cdot \frac{1}{z^{2}} \ldots \ldots \ldots \ldots \ldots \ldots \ldots \ldots \\
& h=\frac{1}{2 \pi}\left\{\left(\alpha_{1}+\alpha_{2}\right)+\sqrt{\alpha_{1} \alpha_{2}}\right\}
\end{aligned}
$$

Neglecting the second order terms with respect to Poisson's ratios, the constants in Eq. (70-a) Eq. (70-d) reduce to

$$
\begin{aligned}
& A=n E_{z} \\
& C=E_{z} .
\end{aligned}
$$




$$
\begin{aligned}
& F=n \nu_{z x} E_{z} \\
& G=\frac{1}{E_{x}}+\frac{1}{E_{z}}+\frac{2 \nu_{z x}}{E_{z}}
\end{aligned}
$$

Substituting Eqs. (74) into (69), and then into Eq. (73) gives

$$
h=\frac{1}{2 \pi}\left\{1+\frac{1}{k}+\frac{1}{k^{2}}+2 \nu_{z x}\left(1-\frac{1}{k^{2}}\right)\right\}
$$

Then, Eq. (72) becomes

$$
\sigma_{z c}=\frac{1}{2 \pi}\left\{1+\frac{1}{k}+\frac{1}{k^{2}}+2 \nu_{z x}\left(1-\frac{1}{k^{2}}\right)\right\} \cdot \frac{1}{z^{2}}
$$

For the value of unit $k$, Eq. (71) and Eq. (76) are known to be identical with isotropic solutions which are usually made use of in stress calculation in soil. These formulae give anisotropic effect on the stress increase in a simpler manner, in term of $k$.

Table 2 Ratio of Vertical Stress Along Load-

\begin{tabular}{|c|c|c|c|c|c|c|c|c|}
\hline \multirow{2}{*}{\multicolumn{3}{|c|}{$\frac{\left(\sigma_{z c}\right) \text { anisotropic }}{\left(\sigma_{z c}\right) \text { isotropic }}$}} & \multicolumn{6}{|c|}{$k=\sqrt{n}$} \\
\hline & & & \multirow{2}{*}{\begin{tabular}{|l|}
1.0 \\
1.00
\end{tabular}} & \multirow{2}{*}{$\frac{0.9}{1.06}$} & \multirow{2}{*}{$\frac{0.8}{1.13}$} & \multirow{2}{*}{\begin{tabular}{|l|}
0.7 \\
1.21
\end{tabular}} & \multirow{2}{*}{\begin{tabular}{|l|}
0.6 \\
1.33
\end{tabular}} & \multirow{2}{*}{$\frac{0.5}{1.50}$} \\
\hline Plane strain & & $J_{p}$ & & & & & & \\
\hline \multirow{3}{*}{$\begin{array}{l}\text { Axial } \\
\text { symmetry }\end{array}$} & \multirow{3}{*}{$J_{t}$} & $\nu=0.00$ & 1.00 & 1.11 & 1.27 & 1.49 & 1.82 & 2.33 \\
\hline & & $\nu=0.25$ & 1.00 & 1.08 & 1.18 & 1.32 & 1.52 & 1.83 \\
\hline & & $\nu=0.50$ & 1.00 & 1.04 & 1.08 & 1.14 & 1.26 & 1.33 \\
\hline
\end{tabular}
ing Line in Anisotropic Solution to that in Isotropic Solution for Two and Three Dimensional Cases.

$J_{p}=(1+1 / k) / 2, \quad J_{t}=\left(1+1 / k+1 / k^{2}+2 \nu\left(1-1 / k^{2}\right)\right) / 3$

For both the two and three dimensional cases, the ratio of the vertical stress along the loading line in the anisotropic solution to that in isotropic solution are calculated in Table 2. From the table, it is known that the influence of $k$ on the stress increase is larger for the axial symmetric case than for the plane strain case, and Poisson's ratio gives an effect to increase the load spreading capacity of the media in the former case.

\section{CONSIDERATIONS ON FRÖHLICH'S STRESS FORMULA}

As is well known, the major principal stress (the radial stress) in incompressible, homogeneous, semi-infinite elastic media which is produced by a unit vertical point load at surface is written as,

$$
\left.\begin{array}{l}
\sigma_{\nu}=\frac{3}{2 \pi z^{2}} \cos ^{3} \theta \\
\tau_{r \theta}=0, \quad \sigma_{\theta}=0
\end{array}\right\}
$$

Considering the deviations of real sand mass from the ideal elastic solid, Fröhlich wrote Eq. (77) as follow by replacing the exponent of the factor $\cos ^{3} \theta$ by an arbitraly exponent $\bar{\nu}$ as

$$
\left.\begin{array}{l}
\sigma_{r}=\frac{\bar{\nu}}{2 \pi z^{2}} \cos ^{\bar{\nu}} \theta \\
\tau_{r \theta}=0, \quad \sigma_{\theta}=0
\end{array}\right\}
$$

The value $\bar{\nu}$ has been called the concentration index (or factor) because it determines the intensity of the pressure on horizontal sections beneath a given point load.

For a vertical unit line load at surface, the radial stress can be obtained by the integration of Eq. (78) as,

$$
\begin{aligned}
& \sigma_{r}=\bar{f}\left(\frac{\cos \theta}{r}\right) \cos ^{\bar{\nu}-3} \theta \ldots . \\
& \bar{f}=\frac{\Gamma\left(\frac{\bar{\nu}+1}{2}\right)}{\sqrt{\pi} \Gamma\left(\frac{\bar{\nu}}{2}\right)} \ldots \ldots \ldots . .
\end{aligned}
$$

Ohde introduced the elastic soil mass in which the stiffness $E$ varies in the manner of

$$
E \propto z^{\kappa}
$$

where $\kappa$ is a constant. He obtained the following relation when the soil behaves as the work done becomes minimum,

$$
\bar{\nu}=3+\kappa
$$

He also showed that the Fröhlich's formula satisfied the strain compatibility equation only in case of

$$
\bar{\nu}=\frac{1}{\nu}+1
$$

where $\nu$ is the isotropic Poisson's ratio. It is is known that for example, in the case that $\kappa=$ $1, \nu=1 / 3, \bar{\nu}=4$ can be assumed for a soil, the Frölich's formula becomes the exact stress solution in elastic media.

Now, express the stresses in the cross-anisotropic mass by the polor coordinates (Fig. 6). Eq. (51) can be transformed to

$$
\left.\begin{array}{l}
\sigma_{r}=\frac{1}{\pi}\left(1+\frac{1}{k}\right)\left(\frac{\cos \theta}{r}\right) \frac{1}{\cos ^{2} \theta+\frac{\sin ^{2} \theta}{k^{2}}} \\
\tau_{r \theta}=0, \quad \sigma_{\theta}=0
\end{array}\right\}
$$

This shows that the stress in the cross-anisotropic mass also takes the radial distribution under 
plane strain condition. For the three dimensional case, Barden stated that the radial distribution of stress can be reasonably assumed, as an engineering approximation, for relatively low degree of anisotropy $(0.5<n<1)$.

From these stated above, usefulness of Fröhlich's formula for sand is obvious.

Then the Fröhlich's formula is used for fitting the tested data on stress distributions, it can be considered that the anisotropy of sand may contribute to the value of the concentration index $\bar{\nu}$. For convenience, the constants in Eqs. (79) and (84) are shown in Table 3 in case of $\theta=0$.

Table 3 The Values of $(1+1 / k) / \pi$ and $\bar{f}$ for $\theta=0^{\circ}$.

\begin{tabular}{c|c|c|c|c|c|c}
\hline$k$ & 1.0 & 0.9 & 0.8 & 0.7 & 0.6 & 0.5 \\
\hline$(1+1 / k) / \pi$ & 0.64 & 0.67 & 0.72 & 0.77 & 0.85 & 0.95 \\
\hline \hline $\bar{y}$ & 3 & 4 & 5 & 6 \\
\hline $\bar{f}$ & 0.64 & 0.75 & 0.85 & 0.94 \\
\hline
\end{tabular}

The meaning of the concentration index is still ambiguous. Because the stress distribution feature in sand can be considered being affected not only by the increase of stiffness with depth, the anisotropic nature of sand but also by a number of factors such as elastic-plastic behaviours of sand, rigidity of footing, surface roughness of footing, loading intensity, geometry of load, etc.

\section{CONCLUSIONS}

(1) It is supposed that for sand the deformation modulus ratio $n$ will be less than unity in common case.

(2) The availability of the anisotropic stressstrain laws for dilatant soil becomes clear.

(3) The stress increase near the load in sand mass can be also explained in terms of the cross anisotropy of the type given by $n<1$. The author considers that the anisotropy is not likely to be the entire explanation for the problem, however, it plays an important role for accerelating the increase of stress and also settlement near the load.

(4) The author's stress solution in plane strain case can be known not to be coincidence with Wolf's.

(5) The ratio $n$ is predominantly important among the momuli in the present problems.

(6) The axial symmetric case has larger effect of the anisotropy than the plane strain case in the vertical stress formula.
(7) The well known Fröhlich's stress formula can be applied to stress problems in the crossanisotropic media, because the cross anisotropic media forms a radial stress field.

(8) The anisotropy of sand sample will have a significant meaning in comparing the data of the triaxial compression test with that of the triaxial extension test.

\section{ACKNOWLEDGEMENT}

The author would like to acknowledge the continuing guidance and encouragement of Prof. F. Kawakami, Tohoku Univ. $\mathrm{He}$ also would like to thank Prof. M. Satake for a helpfull teach for his question. Parts of this paper were presented to Prof. S. Kuranishi as a report for the required subject of the doctor course at the Civil Engineering Dept., Tohoku Univ. The author wishes. to express his gratitude to Dr. Kuranishi for a critical reading of the presented material.

\section{REFERENCES}

1) Arther, J. R. F. and B. K. Menzies (1972): "Inherent anisotropy in a sand," Geot., Vol. 22, No. 1, pp. 115-128.

2) Barden, L. (1963): "Stress and displacement in a cross anisotropic soil," Geot., Vol. 12, No. 3, pp. 198-210.

3) Borowicka, H. (1943): “Die Druckausbreitung im Halbraum bei linear zunehmenden. Elastizitatsmodul," Ing. Archiv., Vol. 14, No. 2 , pp. $75-82$.

4) Curtis, A. J. and F. E. Richard (1955): "Photoelastic analogy for nonhomogeneous foundation," Trans., ASCE, Vol. 120, No. 35.

5) El-Shoby, M. A. (1969): “Deformation of sands under constant stress ratios, Proc., 7th., ICSM, Mexico, pp. 111-119.

6) Hasegawa, T. and T. Sawada (1971): "An. approximate solution of stress distribution. in a heterogeneous and non-linear foundation. and its characteristics," Trans. JSIDRE Feb., pp. 61-70.

7) Ichihara, M. (1955): “On the distribution. of vertical pressure through sand fills caused by rigid loads," Jour., JSCE, Vol. 40, No. 4 (in Japanese).

8) Karst, H., J. Lwgrad, Le Tirant, J. P. Sada and J. Weber (1961): “Contribution a l'Etude de la Mechanique des Milieux Granulaires," Proc. 6th. ICSM, Vol. 1, pp. 259-263.

9) Moroto, N. (1972): "Recoverable deformation of sand," Proc., JSSM, Vol. 12, No. 3, Sept., pp. 65-74 (in Japanese). 
10) Oda, M. and H. Kazama (1970): "Fundamental study on anisotropy of sand," Jour., JSSM, Vol. 18, No. 9, pp. 15-21 (in Japanese).

11) Oda, M. (1972): “The mechanism of fabric changes during compressional deformation of sand," Proc., JSSM, Vol. 12, No. 2, June, pp. 1-17.

12) Ohde, J. (1936): “Zur Theorie der Druckverteilung im Baugrund," Der Bauing., Vol. 25, August, pp. 451-459.

13) Parkin, A. K., C. M. Gerrard, D. R. Willoughly (1968): "Deformation of sand in hydrostatic compression," discussion by Parkin, et al., Jour., SM Div., ASCE, Vol. 94, SM1, pp. 336-340.

14) Pickering, D. J. (1970): “Anisotropic elastic parameters for soil," Geot., Vol. 20, No. 3, pp. 271-276.

15) Rowe, P. W. (1962): “The stress-dilatancy relation for static equilibrium of an assembly of particles in contact," Proc., Roy., Soc., London, A. 269, pp. 500-527.

16) Sobotoka, Z. (1964): "Rhéologie des problèmes de déformation plane des milieux continus," IUTAM Symposium on Rheology and Soil Mech., Grenoble, pp. 218-230.

17) Turnbul, W. J., A. A. Maxwell and R. G. Ahlvin (1961): "Stress and deflections in homogeneous soil masses," Proc., 5th., ICSM, Paris, pp. 337-345.

18) Wolf, K. (1935): “Ausbreitung der kraft in der halbene und im halbraum bei anisotropen material," Zeit. angew. Math. u. Mech., 15, pp. 249-254.

\section{MAIN NOTATIONS}

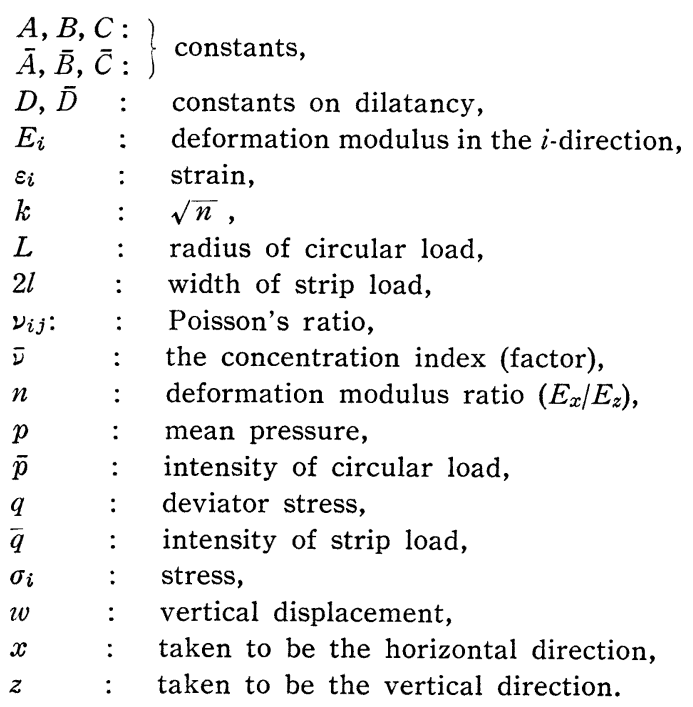

(Received Oct. 7, 1972) 